\title{
The mineralogy of the Ilímaussaq intrusion
}

\author{
Henning Sørensen, John Rose-Hansen and Ole V. Petersen
}

Reviews of the mineralogy of Ilímaussaq have been published by Bøggild $(1905,1953)$, Sørensen (1967) and Semenov (1969).

The lists of minerals and publications have expanded considerably during the ten years which have gone by since these reviews.

It has therefore been felt timely to present a new review of the mineralogy of Ilímaussaq. This review consists of two parts:

I. A list of all minerals identified up to the end of 1979. II. An updated list of all published and planned papers in the series 'Contributions to the Mineralogy of Ilímaussaq'.

A monographic presentation of the mineralogy of the Ilímaussaq intrusion is planned and it is hoped to produce it in the not too distant future.

\section{References}

Bøggild, O. B. 1905: Mineralogia Groenlandica. Meddr Grønland 32, 625 pp.

Bøggild, O. B. 1953: The mineralogy of Greenland. Meddr Grønland 149,3, 422 pp.

Semenov, E. I. 1969: The mineralogy of the Ilimaussaq alkaline massif (in Russian). Moscow: Nauka, $165 \mathrm{pp}$.

Sørensen, H. 1967: On the history of exploration of the Ilímaussaq alkaline intrusion, South Greenland.

Bull. Grønlands geol. Unders. 68 (also Meddr Grønland 181,3), 33 pp.

H.S., J.R-H.,

Institut for Petrologi,

University of Copenhagen,

$\emptyset$ ster Voldgade 10,

DK-1350 Copenhagen $K$.
O.V.P.

Geologisk Museum,

Øster Voldgade 5-7, DK-1350 Copenhagen $K$. 


\section{Minerals from the Ilimaussaq intrusion, South Greenland}

$\begin{array}{ll}\text { NATIVE ELEMENTS } & \\ \text { Graphite } & \mathrm{C} \\ \text { Native antimony } & \mathrm{Sb} \\ \text { Native copper } & \mathrm{Cu} \\ \text { Native lead } & \mathrm{Pb} \\ \text { Native silver } & \mathrm{Ag} \\ \text { Native tin (?) } & \mathrm{Sn}\end{array}$

SULPHIDES

Argentite

'Blaubleibender covellite'

Chalcocite

Chalcopyrite

Chalcostibite

Chalcothallite

Covellite

Digenite

Djerfisherite

Djurleite (?)

Famatinite

Galena

Marcasite

Molybdenite

Pearceite (?)

Polybasite

Pyrite

Pyrrhotite

Rohaite

Skinnerite

Sphalerite

Stannite (?)

Tetrahedrite

Thalcusite

Troilite

Vrbaite (?)

SULPHATES

Beudantite

Brochantite

Connellite

Linarite

SILICATES
Acmite
Aegirine
Aegirine-hedenbergite
Aenigmatite
Albite
Allanite
Analcime
Antigorite
Apophyllite
Arfvedsonite
Astrophyllite
Augite
Bertrandite
Beryllite
Biotite
Britholite
Cancrinite
Catapleiite
Chabazite
Chkalovite

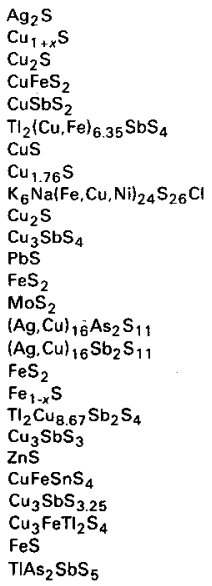

$\mathrm{NaFe}^{+3} \mathrm{Si}_{2} \mathrm{O}_{6}$

(Na. $\mathrm{Ca})\left(\mathrm{Fe}^{+3}, \mathrm{Fe}^{+2}\right)\left\lfloor\mathrm{Si}_{2} \mathrm{O}_{6}\right\rfloor$

(Ca, $\left.\mathrm{Na}, \mathrm{Fe}^{+2}, \mathrm{Fe}^{+3}\right)_{2}\left(\mathrm{Si}_{2} \mathrm{O}_{6}\right)$

$\mathrm{Na}_{2} \mathrm{Fe}_{5}^{2} \mathrm{Til}_{2} \mathrm{O}_{2} \mid \mathrm{Si}_{6} \mathrm{O}_{18}$

$\mathrm{Na} \mid \mathrm{AISi}_{3} \mathrm{O}_{8}$

$\left(\mathrm{Ca}, \mathrm{Ce}_{2}\left(\mathrm{Fe}^{+2}, \mathrm{Fe}^{+3}\right) \mathrm{Al}_{2}|\mathrm{O}| \mathrm{OH}\left|\mathrm{SiO}_{4}\right| \mathrm{Si}_{2} \mathrm{O}_{7} \mid\right.$

$\left.\mathrm{Na} \mid \mathrm{AlSi}_{2} \mathrm{O}_{6}\right\} \cdot \mathrm{H}_{2} \mathrm{O}$

$\mathrm{Mg}_{6}(\mathrm{HOH})_{8} \mid \mathrm{Si}_{4} \mathrm{O}_{10} \mathrm{O}$

$\left.\mathrm{KCa}_{4} \mid \mathrm{F}_{\mid} \mathrm{Si}_{4} \mathrm{O}_{10}\right)_{2} \mid \cdot 8 \mathrm{H}_{2} \mathrm{O}$

$\mathrm{Na}_{3} \mathrm{Fe}_{4}{ }^{2} \mathrm{Fe}^{+3} \mathrm{Si}_{8} \mathrm{O}_{22}\left(\mathrm{OH}_{2}\right.$

(K.Na) ${ }_{3}(\mathrm{Fe}, \mathrm{Mn})_{7}\left(\mathrm{Ti}, \mathrm{Zr}_{2}\left|\mathrm{Si}_{8}(\mathrm{O}, \mathrm{OH})_{31}\right|\right.$

$\left(\mathrm{Ca}, \mathrm{Mg}, \mathrm{Fe}^{+2}, \mathrm{Fe}^{+3}, \mathrm{Ti}, \mathrm{Al}\right)_{2}\left|(\mathrm{Si}, \mathrm{Al})_{2} \mathrm{O}_{6}\right|$

$\left.\mathrm{Be}_{4}\left|(\mathrm{OH})_{2}\right| \mathrm{Si}_{2} \mathrm{O}_{7}\right\}$

$\left.\mathrm{Be}_{3}\left|(\mathrm{OH})_{2}\right| \mathrm{SiO}_{4}\right\} \cdot \mathrm{H}_{2} \mathrm{O}$

$\mathrm{K}(\mathrm{Fe}, \mathrm{Mn})_{3}\left|\mathrm{LOH}, \mathrm{F}_{2}\right| \mathrm{AlSi}_{3} \mathrm{O}_{10}$

$\{\mathrm{Na} . \mathrm{Ce} . \mathrm{Ca})_{5}\left\{\mathrm{~F}\left|\left(\mathrm{SiO}_{4}, \mathrm{PO}_{4}\right)_{3}\right|\right.$

$\left.\mathrm{Na}_{6} \mathrm{Ca}\left\{\mathrm{CO}_{3} \mid(\mathrm{AlSiO})_{4}\right\}_{6}\right\} \cdot 2 \mathrm{H}_{2} \mathrm{O}$

$\left.\mathrm{Na}_{2} \mathrm{ZriSi}_{3} \mathrm{O}_{9}\right\} \cdot 2 \mathrm{H}_{2} \mathrm{O}$

$(\mathrm{Ca}, \mathrm{Na})\left|\mathrm{Al}_{2} \mathrm{Si}_{4} \mathrm{O}_{12}\right| \cdot 6 \mathrm{H}_{2} \mathrm{O}$

$\mathrm{Na}_{2}\left\{\mathrm{BeSi}_{2} \mathrm{O}_{6}\right\}$

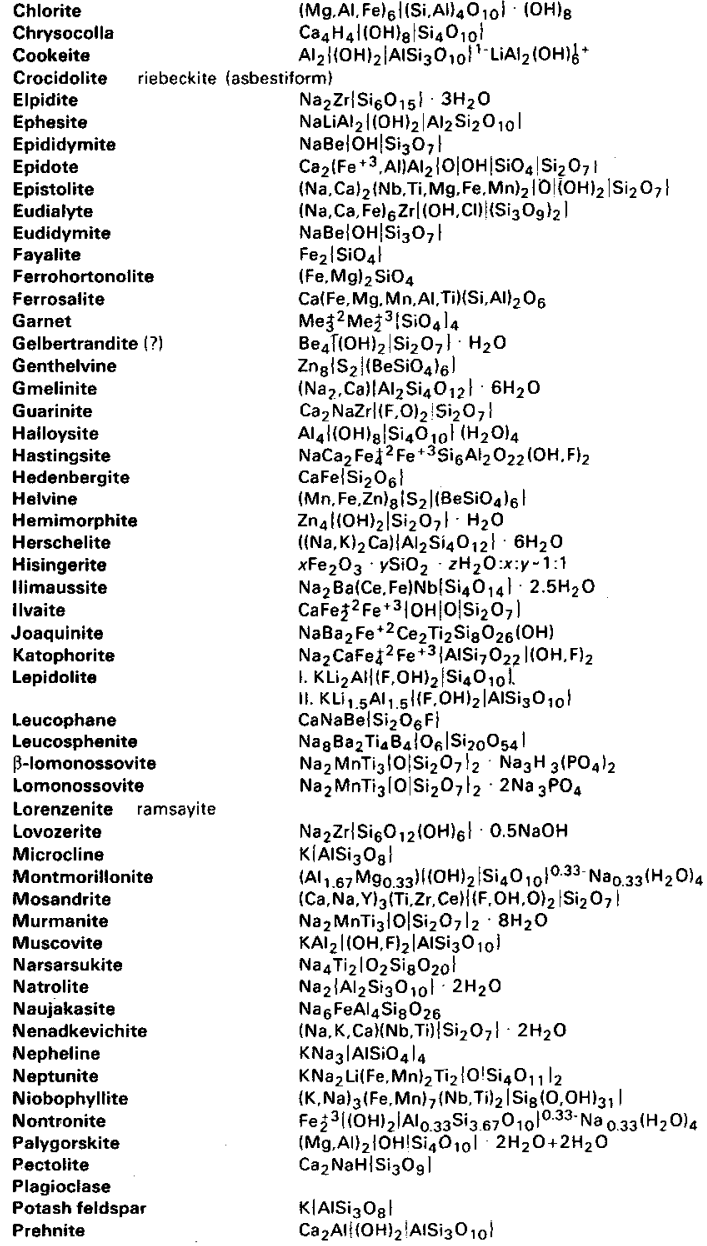

$\mathrm{Na}_{2} \mathrm{Ti}_{2}\left|\mathrm{O}_{3}\right| \mathrm{Si}_{2} \mathrm{O}_{6} \mid$

$\mathrm{Na}_{2} \mathrm{Fe}_{3}^{+2} \mathrm{Fe}_{2}^{3}{ }^{3} \mathrm{Si}_{8} \mathrm{O}_{22}\left(\mathrm{OH}, \mathrm{F}_{2}\right.$

$\mathrm{Na}{ }_{2} \mathrm{Ca}\langle\mathrm{Ca}, \mathrm{Ce})_{4}(\mathrm{Ti}, \mathrm{Nb})\left[(\mathrm{F}, \mathrm{O})_{2}: \mathrm{Si}_{2} \mathrm{O}_{7}\right\}_{2}$

Minkite

Rinkolite = mosandrite

Rosenbuschite

Sauconite

Schizolite pectolite (manganoa

Semenovite

Sepiolite

Serandite

Sodalite

Sorensenite

Spherobertrandite
(Ca Nalo $\mathrm{Zr}\left(\mathrm{Ti} \mathrm{MnN} \mathrm{Nb}\right.$ HF $\mathrm{Ol}_{2} \mathrm{Si}_{2} \mathrm{O}_{7}$ $\left.(\mathrm{Zn}, \mathrm{Mg})_{3}(\mathrm{OH})_{2} \mathrm{iSi}, \mathrm{Al}\right)_{4} \mathrm{O}_{10} \mid(\mathrm{O} .5 \mathrm{Ca}, \mathrm{Na})_{x}\left(\mathrm{H}_{2} \mathrm{O}\right)_{4}$

$\left(\mathrm{Fe}{ }^{+2}, \mathrm{Mn}, \mathrm{Zn}, \mathrm{Ti}\right) \mathrm{RE}_{2} \mathrm{NaO}_{2}(\mathrm{Ca}, \mathrm{Na})_{8}(\mathrm{Si}, \mathrm{Be})_{20}\left(\mathrm{O}, \mathrm{OH}_{1} \mathrm{~F}_{48}\right.$ $\mathrm{Mg}_{4}\left|(\mathrm{OH})_{2} \mathrm{Si}_{6} \mathrm{O}_{15}\right| \cdot 2 \mathrm{H}_{2} \mathrm{O}+4 \mathrm{H}_{2} \mathrm{O}$

$\left(\mathrm{Mn}+2, \mathrm{Ca}_{2} \mathrm{NaH} \mathrm{Si}_{3} \mathrm{O}_{9}\right.$

$\mathrm{Na}_{8} \mid \mathrm{Cl}_{2}\left(\mathrm{AlSiO}_{4}\right)_{6}$

$\mathrm{Na}_{4} \mathrm{SnBe}_{2} \mid(\mathrm{OH})_{4} \mathrm{Si}_{6} \mathrm{O}_{16}$

$\mathrm{Be}_{5} \mid(\mathrm{OH})_{4}: \mathrm{Si}_{2} \mathrm{O}_{7}$ 
Steenstrupine

Stilbite

Stillwellite

Tetranatrolite

Thorite

Titanite

Tugtupite

Tundrite

Uranothorite

Ussingite

Vesuvianite

Willemite

Zircon

\section{CARBONATES}

Azurite (?)

Bastnaesite

Calcite

Cerussite

Connellite

Hydrocerussite

Malachite (antimonian)

Nahcolite

Siderite

Synchysite

Thermonatrite

Trona
$\mathrm{Na}{ }_{12} \cdot{ }_{x} \mathrm{H}_{x} \mathrm{Ca}(\mathrm{La}, \mathrm{Ce}, \mathrm{Nd})_{6}(\mathrm{Mn}, \mathrm{Fe}, \mathrm{Th}, \mathrm{Zr}, \mathrm{U})_{5}\left(\mathrm{Si}{ }_{6} \mathrm{O}_{18}\right\}_{2}\left\{\left.(\mathrm{P}, \mathrm{Si}) \mathrm{O}_{4}\right|_{6}(\mathrm{OH}, \mathrm{Cl}) \cdot n \mathrm{H}_{2} \mathrm{O}\right.$

$\mathrm{Ca}\left|\mathrm{Al}_{2} \mathrm{Si}_{7} \mathrm{O}_{18}\right| 7 \mathrm{H}_{2} \mathrm{O}$

$\mathrm{CeB}\left|\mathrm{O} \mathrm{SiO}_{4}\right|$

$\mathrm{Na}_{2}\left|\mathrm{Al}_{2} \mathrm{Si}_{3} \mathrm{O}_{10}\right| \quad 2 \mathrm{H}_{2} \mathrm{O}$

$\mathrm{Th}^{\prime}\left|\mathrm{SiO}_{4}\right|$

CaTilO $/ \mathrm{SiO}_{4} i$

$\left.\mathrm{Na}_{9}\left|\mathrm{Cl}_{2}\right|\left(\mathrm{BeA}_{1} \mid \mathrm{Si}_{4} \mathrm{O}_{12}\right)_{2}\right)$

$\mathrm{Na}_{2} \mathrm{Ce}_{2} \mathrm{Ti} \mathrm{O}_{4}\left\lceil\mathrm{SiO}_{4} \mid \quad 4 \mathrm{H}_{2} \mathrm{O}\right.$

$\left(\mathrm{Th}^{\mathrm{U}}\right)^{\mathrm{SiO}} \mathrm{Si}_{4}$

$\mathrm{Na}_{2}\left|(\mathrm{OH}): \mathrm{AlSi}_{3} \mathrm{O}_{8}\right|$

$\mathrm{Ca}_{10}\left(\mathrm{Mg} . \mathrm{Fe}_{2} \mathrm{Al}_{4}\left|(\mathrm{OH})_{4}\right|\left(\mathrm{SiO}_{4}\right)_{5} \mid\left(\mathrm{Si}_{2} \mathrm{O}_{7}\right)_{2}\right\}$

$\mathrm{Zn}_{2} \mid \mathrm{SiO}_{4}$

$\left.\mathrm{Zr} \mid \mathrm{SiO}_{4}\right\}$

$\mathrm{Cu}_{3}\left(\mathrm{OH} / \mathrm{CO}_{3}\right)_{2}$

$\mathrm{Ce}|\mathrm{F}| \mathrm{CO}_{3} \mid$

$\mathrm{CaCO}_{3}$

$\mathrm{PbCO}_{3}$

$\left.\mathrm{Cu}_{1} / \mathrm{Cl}_{4} \mid \mathrm{OH}\right)_{32} / \mathrm{SO}_{4}+4 \mathrm{H}_{2} \mathrm{O}$

$\left.\mathrm{Pb}_{3}|\mathrm{OH}| \mathrm{CO}_{3}\right|_{2}$

$\mathrm{Cu}_{2}\left|(\mathrm{OH})_{2}\right| \mathrm{CO}_{3}$

$\mathrm{NaHCO}_{3}$

$\mathrm{FeCO}_{3}$

$\left.\mathrm{CaCe}|\mathrm{F}| \mathrm{CO}_{3}\right)_{2} \mid$

$\mathrm{Na}_{2} \mathrm{CO}_{3} \cdot \mathrm{H}_{2} \mathrm{O}$

$\mathrm{Na}_{3} \mathrm{H}\left(\mathrm{CO}_{3}\right)_{2} \cdot 2 \mathrm{H}_{2} \mathrm{O}$
OXIDES

Avicennite (?) $\quad \mathrm{Tl}_{2} \mathrm{O}_{3}$

$\left(\mathrm{Cu}, \mathrm{Sb}_{2}\left(\mathrm{~Pb},(\mathrm{Fe}, \mathrm{Ca}) \mathrm{Si}_{\mathrm{O} .4}\left(\mathrm{O}, \mathrm{OH}, \mathrm{H}_{2} \mathrm{O}\right.\right.\right.$

Chalcedony $\mathrm{SiO}_{2}$

Cuprite $\quad \mathrm{Cu}_{2} \mathrm{O}$

AlOOH

Gerasimovskite $\quad \mathrm{TiNb}(\mathrm{OH})$

$\begin{array}{ll}\text { Hematite } & \mathrm{Fe}_{2} \mathrm{O}_{3} \\ \text { Hydrargillite } & \text { AllOH) }\end{array}$

Igdloite = lueshite

Ilmenite $\quad \mathrm{FeTiO}_{3}$

Limonite $\quad \mathrm{Fe}_{2} \mathrm{O}_{3} \cdot 1.5 \mathrm{H}_{2} \mathrm{O}$

Litharge $\quad \mathrm{PbO}_{2}$

Lueshite $\quad \mathrm{NaNbO}_{3}$

Magnetite $\quad \mathrm{Fe}_{3} \mathrm{O}$

Plattnerite $\quad \mathrm{PbO}$

Pyrochlore $\quad(\mathrm{Na}, \mathrm{Ca})_{2}(\mathrm{Nb}, \mathrm{Ta})_{2} \mathrm{O}_{6}(\mathrm{O}, \mathrm{OH}, \mathrm{F})$

Pyrolusite $\mathrm{MnO}_{2}$

Pyrophanite $\mathrm{MnTiO}_{3}$

Quartz $\quad \mathrm{SiO}_{2}$

Rutile $\quad \mathrm{TiO}_{2}$

$\begin{array}{ll}\text { Senarmontite } & \mathrm{Sb}_{2} \mathrm{O}_{3} \\ \text { Thorianite } & \text { (Th, U iO }\end{array}$

Titanomagnetite

Todorokite $\left(\mathrm{Mn}^{* 4}, \mathrm{Mn}^{2}{ }^{2}\right)_{8}(\mathrm{O}, \mathrm{OH})_{16}+2 \mathrm{H}_{2} \mathrm{O}$

Valentinite

$\mathrm{Sb}_{2} \mathrm{O}_{3}$

\begin{tabular}{|c|c|c|c|}
\hline ARSENIDES AN & NIDES & PHOSPHATES & \\
\hline Allargentum & AgSb & Apatite & $\mathrm{Ca}_{5}\left[(\mathrm{~F}, \mathrm{OH})\left(\mathrm{PO}_{4}\right\rfloor_{3} \mid\right.$ \\
\hline Breithauptite & $\mathrm{NiSb}$ & Britholite & $(\mathrm{Na} . \mathrm{Ce}, \mathrm{Ca})_{5} \mid \mathrm{F}\left(\mathrm{SiO}_{4}, \mathrm{PO}_{4}\right)_{3}$ \\
\hline Cuprostibite & $\mathrm{Cu}_{2} \mathrm{Sb}$ & Carbonate hydroxylapatite & $\mathrm{Ca}_{5}\left[(\mathrm{~F}, \mathrm{OH}, \mathrm{O})\left(\mathrm{PO}_{4}, \mathrm{CO}_{3}\right)_{3}\right\}$ \\
\hline Dyscrasite & $\mathrm{Ag}_{3} \mathrm{Sb}$ & Dahllite carbonare hydroxy & dapatite \\
\hline Gudmundite & FeSos & Monazite & $\left.\mathrm{Ce}_{\mathrm{PO}}\right\}$ \\
\hline Loellingite & $\mathrm{FeAs}_{2}$ & Rhabdophane & (Ce.La)PO $\mathrm{P}_{4} \quad \mathrm{H}_{2} \mathrm{O}$ \\
\hline Niccolite & $\mathrm{NiAs}$ & Vitusite & $\mathrm{Na}_{3}(\mathrm{Ce}, \mathrm{La})\left(\mathrm{PO}_{4}\right)_{2}$ \\
\hline Seinäjokite & $\mathrm{FeSb}_{2}$ & & \\
\hline Skutterudite & $\mathrm{CoAs}_{3}$ & & \\
\hline Vystervejoute & (FE, NI, ColAS & SOLID HYDROCARBONS & \\
\hline & & Evenkite-like & $\mathrm{C}_{22} \mathrm{H}_{46}-\mathrm{C}_{33} \mathrm{H}_{68}$ \\
\hline HALOGENIDES & & UNNAMED & \\
\hline $\begin{array}{l}\text { Fluorite } \\
\text { Villiaumite }\end{array}$ & $\mathrm{CaF}_{2}$ & & $\begin{array}{l}\mathrm{Na}(\mathrm{Ce}, \mathrm{Ca})_{9} \mathrm{Si}_{6} \mathrm{O}_{26} \\
\mathrm{Na}_{4} \mathrm{TiNb}_{2} \mathrm{Si}_{4} \mathrm{O}_{17} \cdot 2 \mathrm{Na}_{2} \mathrm{H}\left(\mathrm{PO}_{4}\right)\end{array}$ \\
\hline
\end{tabular}

The chemical formulae of the minerals are taken from Strunz (1970) except when newer data are available.

Reference. Strunz, H. 1970: Mineralogische Tabellen, 621 pp. Leipzig: Akademische Verlagsgesellschaft. Geest \& Portig K.-G. 


\section{Contributions to the mineralogy of llimaussaq}

No. 1 Semenov,E.I., Gerassimovsky,V.I., Maksimova,N.V., Andersen,S. \& Petersen,O.V. 1965: Sorensenite, a new sodium-beryllium-tin-silicate from the Ilimaussaq intrusion, South Greenland. Bull. Gronlands geol. Unders. 61 (also Meddr Grønland 181,1) $19 \mathrm{pp}$.

No. 2 Semenov,E.I. \& Sørensen,H. 1966: Eudidymite and epididymite from the llimaussaq alkaline intrusion, South Greenland. Bull. Grønlands geol. Unders. 63 (also Meddr Grønland 181,2) 21 pp.

No. 3 Sørensen,H. 1967: On the history of exploration of the llimaussaq alkaline intrusion, South Greenland. Bull. Grønlands geol. Unders. 68 (also Meddr Grønland 181,3) 33 pp.

No. 4 Bollingberg,H. \& Petersen,O.V. 1967: Genthelvite from the llimaussaq alkaline intrusion, South Greenland. Bull. Grøn/ands geol. Unders. 68 (also Meddr Grønland 181,4) 1-9.

No. 5 Andersen,S. 1967: On beryllite and bertrandite from the llimaussaq alkaline intrusion, South Greenland. Bull. Gronlands geol. Unders. 68 (also Meddr Grønland 181,4) 11-27.

No. 6 Semenov,E.I., Kazakova,M.E. \& Aleksandrova,R.A. 1967: The Lovozero minerais-nenadkevichite, gerassimovskite and tundrite - from Ilimaussaq, South Greenland. Bull. Grønlands geol. Unders. 68 (also Meddr Grønland 181,5) 1-11.

No. 7 Semenov,E.I., Sørensen,H., Bessmertnaja,M.S \& Novorossova,L.E. 1967: Chalcothallite - a new sulphide of copper and thallium from the Ilimaussaq alkaline intrusion, South Greenland. Bull. Grøn/ands geol. Unders. 68 (also Meddr Grønland 181,5) 13-26.

No. 8 Petersen,O.V. 1966: Crossed axial plane dispersion in epididymite. Amer. Miner. 51, 916-919.

No. 9 Petersen,O.V. 1967: The mineralogy of naujakasite. Bull. Grønlands geol. Unders. 75 (also Meddr Grønland 181,6) $17 \mathrm{pp}$.

No.10 Semenov,E.I., Kazakova,M.E. \& Bukin,V.J. 1968: llimaussite, a new rare earth-niobium-barium silicate from Ilimaussaq, South Greenland. Bull. Grønlands geol. Unders. 75 (also Meddr Grønland 181,7) 3-7.

No.11 Semenov,E.I., Sørensen,H. \& Katajeva,Z.T. 1968: On the mineralogy of pyrochlore from the Ilimaussaq alkaline intrusion, South Greenland. Bull. Gronlands geol. Unders. 75 (also Meddr Grønland 181,7) 9-24.

No.12 Hansen,J. 1968: A study of radioactive veins containing rare-earth minerals in the area surrounding the lifmaussaq alkaline intrusion in South Greenland. Bull. Grønlands geol. Unders. 76 (also Meddr Grønland 181,8) 47 pp.

No.13 Andersen,E.K., Danø,M. \& Petersen,O.V. 1969: A tetragonal natrolite. [from the llimaussaq alkaline intrusion, South Greenland.] Bull. Grønlands geol. Unders. 79 (also Meddr Grønland 181,10) 19 pp.

No.14 Sørensen,H., Hansen,J. \& Bondesen,E. 1969: Preliminary account of the geology of the Kvanefjeld area of the llimaussaq intrusion, South Greenland. Rapp. Gronlands geol. Unders. 18, $40 \mathrm{pp}$.

No.15 Sobolev,V.S., Bazarova,T.Y., Shugurova,N.A., Bazarov,L.Sh., Dolgov,Yu.A. \& Sørensen,H. 1970: A preliminary examination of fluid inclusions in nepheline, sorensenite, tugtupite and chkalovite from the Ilimaussaq alkaline intrusion, South Greenland. Bull. Grønlands geol. Unders. 81 (also Meddr Grønland 181,11) $32 \mathrm{pp}$.

No.16 Semenov,E.I., Bukin,V.I., Balashov,Yu.A. \& Sørensen,H. 1967: Rare earths in minerals of the joaquinite group. Amer. Miner. 52, 1762-1769.

No.17 Sørensen,H., Leonardsen,E.S. \& Petersen,O.V. 1970: Trona and thermonatrite from the llimaussaq alkaline intrusion, South Greenland. Bull. geol. Soc. Denmark 20, 1-19.

No.18 Petersilie,I.A. \& Sörensen,H. 1970: Hydrocarbon gases and bituminous substances in rocks from the llimaussaq alkaline intrusion, south Greenland. Lithos 3, 59-76.

No.19 Povarennykh,A.S., Platonov,A.N. \& Belichenko,V.P. 1970: On the colour of ussingite from the llimaussaq (South Greenland) and Lovozero (Kola Peninsula) alkaline intrusions, Bull. geol. Soc. Denmark 20, 20-26.

No.20 Sørensen,H., Danø.M. \& Petersen,O.V. 1971: On the mineralogy and paragenesis of tugtupite $\mathrm{Na}{ }_{8} \mathrm{Al}_{2} \mathrm{Be}_{2} \mathrm{Si}_{8} \mathrm{O}_{24}(\mathrm{Cl}, \mathrm{S})_{2}$ from the Ilimaussaq alkaline intrusion, South Greenland. Bull. Grøn/ands geol. Unders. 95 I (also Meddr Grønland 181,13) $38 \mathrm{pp}$. 
No.21 Povarennykh,A.S., Platonov,A.N., Tarashchan,A.N. \& Belichenko,V.P. 1971: The colour and luminescence of tugtupite (beryllosodalite) from Ilimaussaq, South Greenland. Bull. Gronlands geo/. Unders. 95 II (also Meddr Grønland 181,14) 12 pp.

No.22 Engell,J., Hansen,J., Jensen,M., Kunzendorf,H. \& Løvborg,L. 1971: Beryllium mineralization in the llimaussaq intrusion, South Greenland, with description of a field beryllometer and chemical methods. Rapp. Grønlands geol. Unders. 33, $40 \mathrm{pp}$

No.23 Løvborg,L., Wollenberg,H., Sørensen,P. \& Hansen,J. 1971: Field determination of uranium and thorium by gamma-ray spectrometry, exemplified by measurements in the Ilimaussaq alkaline intrusion, South Greenland. Econ. Geol. 66, 368-384.

No.24 Wollenberg,H., Kunzendorf,H. \& Rose-Hansen,J. 1971: Isotope-excited X-ray fluorescence analyses for $\mathrm{Nb}, \mathrm{Zr}$, and $\mathrm{La}+\mathrm{Ce}$ on outcrops in the llimaussaq intrusion, South Greenland. Econ. Geol. 66 , 1048-1060.

No.25 Bohse,H., Brooks,C.K. \& Kunzendorf,H. 1971: Field observations on the kakortokites of the llimaussaq intrusion, South Greenland, including mapping and analyses by portable X-ray fluorescence equipment for zirconium and niobium. Rapp. Grønlands geol. Unders. 38, $43 \mathrm{pp}$.

No.26 Petersen,O.V. \& Rönsbo,J.G. 1972: Semenovite a new mineral from the llimaussaq alkaline intrusion. south Greenland. Lithos 5, 163-173.

No.27 Cannilo,E., Mazzi,F. \& Rossi,G. 1972: The structure type of joaquinite. Tschermaks miner. petrogr. Mitt. 17, 233-246.

No.28 Engell,J. 1973: A closed system crystal-fractionation model for the agpaitic llimaussaq intrusion, South Greenland with special reference to the lujavrites. Bull. geol. Soc. Denmark 22, 334-362.

No.29 Petersen,O.V. \& Andersen,S. 1975: The crystal habit of naujakasite with note on some new occurrences. Bull. Grønlands geol. Unders. 116, 5-9.

No.30 Sørensen,H., Rose-Hansen,J., Nielsen,B.L., Løvborg,L., Sørensen,E. \& Lundgaard,T. 1974: The uranium deposit at Kvanefjeld, the llimaussaq intrusion, South Greenland. Geology, reserves and beneficiation. Rapp. Gronlands geol. Unders. 60,54 pp.

No.31 Larsen,L.M. \& Steenfelt,A. 1974: Alkali loss and retention in an iron-rich peralkaline phonolite dyke from the Gardar province, south Greenland. Lithos 7, 81-90.

No.32 Karup-Møller,S. 1974: Mineralogy of two copper-antimony-sulphide-oxide occurrences from the llimaussaq alkaline intrusion in South Greenland. Neues Jb. Miner. Abh. 122, 291-313.

No.33 Karup-Møller,S. \& Makovicky,E. 1974: Skinnerite $\mathrm{Cu}_{3} \mathrm{SbS}_{3}$, a new sulfosalt from the llimaussaq alkaline intrusion, South Greenland. Amer. Miner. 59, 889-895.

No.34 López-Soler,A., Bosch-Figueroa,J.M., Karup-Møller,S., Besteiro,J. \& Font-Altaba,M. 1975: Optical study of cuprostibite ( $\left(\mathrm{Cu}_{2} \mathrm{Sb}\right)$. Fortschr. Miner. 52, 557-565.

No.35 Bohse,H., Rose-Hansen,J., Sørensen,H., Steenfelt,A., Løvborg,L. \& Kunzendorf,H. 1974: On the behaviour of uranium during crystallization of magmas - with special emphasis on alkaine magmas. $/ n$ : Formation of uranium ore deposits, 49-60. Vienna: International Atomic Energy Agency.

No.36 Steenfelt,A. \& Bohse,H. 1975: Variations in the content of uranium in eudialyte from the differentiated alkaline llimaussaq intrusion, south Greenland. Lithos 8, 39-45.

No.37 Karup-Møller,S. 1975: On the occurrence of native lead, litharge, hydrocerussite and plattnerite within the llimaussaq alkaline intrusion in South Greenland. Neues Jb. Miner. Mh., 1975, 229-241.

No.38 Larsen,L.M. 1976: Clinopyroxenes and coexisting mafic minerals from the alkaline llimaussaq intrusion, South Greenland. J. Petrology 17, 258-290.

No.39 Platt,R.G. \& Rose-Hansen,J. 1975: The system ussingite-water and its bearing on crystallization in persodic portions of the system $\mathrm{Na}_{2} \mathrm{O}-\mathrm{Al}_{2} \mathrm{O}_{3}-\mathrm{SiO}_{2}-\mathrm{H}_{2} \mathrm{O}$ at $1 \mathrm{~kb}$ total pressure. J. Geol. 83, 763-772.

No.40 Andersen,E.B., Fenger,J. \& Rose-Hansen,J. 1975: Determination of $\left[\mathrm{Fe}^{2+}\right] /\left[\mathrm{Fe}^{3+}\right]$-ratios in arfvedsonite by Mössbauer spectroscopy. Lithos 8, 237-246.

No.41 Blaxland,A.B., van Breemen,O. \& Steenfelt,A. 1976: Age and origin of agpaitic magmatism at llimaussaq, south Greenland: Rb-Sr study. Lithos 9, 31-38.

No.42 Karup-Møller,S. 1976: Gmelinite and herschelite from the llimaussaq intrusion in South Greenland. Mineralog. Mag. 40, 867-873. 
No.43 Petersen, O.V. 1978: The twin formation of tugtupite, a contribution. Mineralog. Mag. 42, 251-254.

No.44 Oen I.S., Burke,E.A.J. \& Kieft,C. 1977: Westerveldite from Igdlúnguaq, Ilimaussaq alkaline massif, South Greenland. Mineralog. Mag. 41, 77-83.

No.45 Karup-Møller,S. \& Makovicky,E. 1977: Westerveldite from the llimaussaq alkaline intrusion in South Greenland. Mineralogy, crystallography, mineral associations and alteration products. Neues $J b$. Miner. Abh. 130, 208-242.

No.46 Karup-Møller,S., Løkkegaard,L., Semenov,E.I. \& Sørensen,H. 1978: The occurrence of cuprostibite. Bull. Grønlands geol. Unders. 126, 7-22.

No.47 Karup-Møller,S. 1978: Primary and secondary ore minerals associated with cuprostibite. Bull. Grøn/ands geol. Unders. 126, 23-47.

No.48 Karup-Møller,S. 1978: The ore minerals of the llimaussaq intrusion: their mode of occurrence and their conditions of formation. Bull. Grønlands geol. Unders. 127, $51 \mathrm{pp}$.

No.49 Larsen,L.M. 1977: Aenigmatites from the llimaussaq intrusion, south Greenland: chemistry and petrological implications. Lithos 10, 257-270.

No.50 Hansen,J.K.G. 1977: Sulphatising roasting of a Greenlandic uranium ore, reactivity of minerals and recovery. Risø Rep. 355, 129 pp.

No.51 Mazzi,F., Ungaretti,L., Dal Negro,A., Petersen,O.V. \& Rönsbo,J.G. 1979: The crystal structure of semenovite. Amer. Miner. 64, 202-210.

No.52 Konnerup-Madsen,J., Larsen,E. \& Rose-Hansen,J. 1979: Hydrocarbon-rich fluid inclusions in minerals from the alkaline Ilimaussaq intrusion, South Greenland. Bull. Minér. 102, 642-653.

No.53 Larsen,J.G. 1977: Petrology of the late lavas of the Eriksfjord Formation, Gardar province, South Greenland. Bull. Grønlands geol. Unders. 125, $31 \mathrm{pp}$.

No.54 Sørensen,H. 1978: The position of the augite syenite and pulaskite in the llimaussaq intrusion, South Greenland. Bull. geol. Soc. Denmark 27, spec. issue, 15-23.

No.55 Nielsen,B.L. \& Steenfelt,A. 1979: Intrusive events at Kvanefjeld in the Ilimaussaq igneous complex. Bull. geol. Soc. Denmark 27, 143-155.

No.56 Larsen,L.M. 1979: Distribution of REE and other trace elements between phenocrysts and peralkaline undersaturated magmas, exemplified by rocks from the Gardar igneous province, south Greenland. Lithos $12,303-315$.

No.57 Rønsbo,J.G., Khomyakov,A.P., Semenov,E.I., Voronkov,A.A. \& Garanin,V.K. 1979: Vitusite - a new phosphate of sodium and rare earths from the Lovozero alkaline massif, Kola, and the llimaussaq alkaline intrusion, south Greenland. Neues $J b$. Miner., Abh. 137, 42-53.

No.58 Makovicky,E., Johan,Z. \& Karup-Møller,S. 1980: New data on bukovite, thalcusite, chalcothallite and rohaite. Neues Jb. Miner., Abh. 138, 122-146.

No.59 Nielsen,B.L. in press: Case history of the exploration of the Kvanefjeld area, South Greenland. / $n$ : Case histories of uranium exp/oration. Vienna: International Atomic Energy Agency.

No.60 Makovicky,E. \& Karup-Møller,S. 1981: Crystalline steenstrupine from Tunugdliarfik in the llimaussaq alkaline intrusion, South Greenland. Neues Jb. Miner., Abh. 140, 300-330.

No.61 Petersen,O.V. 1981: The first natural crystals of NaF, villiaumite. Neues Jb. Miner., Mh. 1981, 111-116.

No.62 Larsen,L.M. 1981: Sector zoned aegirine from the llimaussaq alkaline intrusion, South Greenland. Contr. Min. Petr. 76, 285-291. 